\title{
Non Classical Endosomal Compartments Play Important Roles in Cross-Presentation by Dendritic Cells
}

\author{
Imai J*, Yokoyama Y, Otani M and Sakai T \\ Laboratory of Physiological Chemistry, Faculty of Pharmacy, Takasaki University of \\ Health and Welfare, Takasaki, Gunma 370-0033, Japan
}

*Corresponding author: Jun Imai, Laboratory of Physiological Chemistry, Faculty of Pharmacy, Takasaki University of Health and Welfare, Takasaki, Gunma 370-0033, Japan, Tel: +81-27-352-1180 (8326); Email: jimai@takasaki-u.ac.jp

\section{Abstract}

Major histocompatibility complex class I (MHC I) molecules are expressed on the surface of all nucleated cells associated with short antigenic peptides about ten amino acids long at their extracellular part. The antigenic peptides are derived from endogenous proteins, which are processed by the ubiquitin-proteasome system in the cytosol. In contrast, exogenous proteins are processed by lysosomal proteases and resultant antigenic peptides are presented upon Major histocompatibility complex class II (MHC II) molecules. In several kinds of cells, especially in dendritic cells (DCs), exogenous proteins are processed and presented upon MHC I in addition to MHC II. This phenomenon is called crosspresentation (CP). CP plays important roles not only in activations of CD8 ${ }^{+} \mathrm{T}$ cells into cytotoxic $\mathrm{T}$ lymphocytes (CTLs) against anti-infectious and anti-tumor immunity but also in in-activations of self-acting CD8 ${ }^{+} \mathrm{T}$ cells by $\mathrm{T}$ cell energy or $\mathrm{T}$ cells deletion for peripheral tolerance. To this date, because of the important roles for $\mathrm{CP}$, there are accumulating evidence to elucidate the molecular mechanisms of CP. Recent researches indicated that the endoplasmic reticulum (ER)associated degradation (ERAD) pathway plays a central role in processing of exogenous proteins. The conveyances of exogenous proteins into ERAD-possible compartments are dependent upon the integration among endosomal or phagosomal membrane with ER membrane. But there still remain several important questions about $\mathrm{CP}$, such as the precious molecular mechanisms of these integrations and the peptide-loading mechanisms upon MHC I. This review describes the recent researches about the molecular mechanisms of CP.

Keywords: DC: Dendritic cells; CP: Cross presentation; MHC I: Major histocompatibility complex class I; ER: endoplasmic reticulum; ERAD: Endoplasmic reticulum associated degradation; PLC: Peptide loading complex. 


\section{Open Access Journal of Pharmaceutical Research}

\section{Introduction}

\section{Major Histocompatibility Complexes and Cellular Immunity}

Major histocompatibility complex class I (MHC I) corresponds to the human leukocyte antigen (HLA) -A, -B, and -C are found on the surface of all nucleated cells [1]. However these HLAs are famous for the major cause of organ transplant rejections, they play important roles in disease defense against in-cell parasite bacteria, viruses, and cancers. MHC I bound with antigenic-peptides about ten amino acids long at their extracellular part and displays these antigenic-peptides against CTLs [1]. These antigenic peptides are generated from endogenous proteins by the ubiquitin-proteasome systems. CTLs specifically recognize these MHC I molecules with non-self antigenic peptides by their surface molecular complex called $\mathrm{T}$ cell receptor (TcR) and differentiate self-cells and non-self cells. After ligation of TcR with MHC I with nonself antigenic peptides, CTLs release the cytotoxins (perforin, granzymes, and granulysin) [2] or express the FAS ligand (CD95L) on their surface [3]. Either process eventually leads apoptosis against those non-self cells. In distinction from MHC I, expression of MHC class II (MHC II) is restricted to only professional Antigen-presenting cells (without mentioning described as APC) [4]. APCs have specific abilities to show antigenic peptide from extracellular proteins upon MHC II against T cells. MHC II interacts with $\mathrm{TcR}$ of $\mathrm{CD} 4^{+} \mathrm{T}$ cells and regulates these $\mathrm{CD}^{+} \mathrm{T}$ cells to start appropriate immune responses [4].

MHC I molecules shows high polymorphism and most of their variations are concentrated around peptide binding groove at their extracellular part [1]. This diversity enables MHC I to show a wide variety of antigenic-peptide against immune systems and enables our immune system to react with various diseases. To insure the multiplicity of antigenic peptide, our immune system furnished with splendid molecular apparatus to supply the diversity of antigenic peptide upon MHC I. Antigenic peptides are processed by the ubiquitinproteasome system in the cytosol from endogenous proteins [1] and then are transported into ER trough the transporter associated with antigen processing 1 (TAP1)/TAP2 complex (TAP complex) [5,6]. In ER, antigenic-peptides are inserted into peptide binding groove of newly translated MHC I with the aid of the peptide-loading complex (PLC), which is consistent from Tapasin, Calreticulin, and ERp57 (PDI) other than TAP complex. TAP complex transports a wide variety of peptides into ER, because of their low specificity against substrates [5,6]. In ER, MHC I are connected with TAP complex by Tapasin so as to guarantee the rapid loading of an antigenic peptide. These in no time responses also protect these antigenic peptides from high amino peptidase activity in both cytosol and ER. In addition, these peptide loadings are assisted by specific chaperones, Calreticulin and ERp57 (PDI), which ensure the correct folding of MHC I complex. After binding with an antigenic peptide, the MHC I is released from the PLC and are transported toward the cell surface. These subsequent processes are called direct-presentation (DP) [7].

\section{Cross-Presentation}

CTLs, which destroy cancer cells or infected cells, are derived from $\mathrm{CD}^{+} \mathrm{T}$ lymphocytes. However the number of each antigen-specific CD8 ${ }^{+} \mathrm{T}$ cells is too small, and in addition, they cannot destroy non-self cells before differentiation into CTLs. To revitalize non-activated CD8 ${ }^{+}$ $\mathrm{T}$ cells into CTLs and to proliferate them into enough numbers, two stimulations are indispensable; specific TcR ligations against MHC I complexes loaded with antigenic peptide and $\operatorname{CD} 28$ against co-stimulatory molecules (CD80, CD86) upon APCs [8,9]. Among these two stimulations, the ligation between TcR and MHC I complex determines the specificity of CTL. Since CTLs can only destroy target cells with just the according to antigenic peptides upon MHC I as APCs, APCs would present antigenic peptides derived from specific proteins of infected cells or cancer cells [10]. For this purpose, APCs have a specific ability to take up and process extracellular proteins so as to present those resultant non-self peptides upon their own surface MHC I. These sequential processes are called antigen cross-presentation (CP) and play definitive roles in the decision of the target cells for CTLs [10-13].

In contrast to TcR-MHC I signal, the co-stimulatory signal decides the general direction of differentiation upon $\mathrm{CD}^{+} \mathrm{T}$ cells. Co-stimulatory molecules are expressed only upon activated APCs by innate immunity. When $\mathrm{CD}^{+} \mathrm{T}$ cells are activated by TcR-MHC I signal together with the co-stimulatory signal, $\mathrm{CD}^{+} \mathrm{T}$ cells differentiated into CTLs. These activations are called cross-priming [14]. To the contrary, if $\mathrm{CD}^{+} \mathrm{T}$ cells are activated by TcR-MHC I signal by steady state APC without the co-stimulatory signal, $\mathrm{CD}^{+} \mathrm{T}$ cells fall into energy or are led into apoptosis. Those in activations are designated as cross-tolerance [14-17].

\section{Dendritic Cells}

APCs include macrophages, B cells, and dendritic cells. They show specialized ability in presenting antigen to $\mathrm{T}$ cells. They also express a set of pattern recognition molecules for discrimination of kinds of non-self in their 


\section{Open Access Journal of Pharmaceutical Research}

environments to decide the activations and direction of acquired immunity. Among above three APCs, DCs show the strongest ability of CPs. The name of DC is derived from their arboroid branch structure (dendrite) found in draining lymph node. DCs, kinds of hematopoietic bone marrow progenitor cells, found in the almost whole of our body. Immature DCs continuously explore the surrounding environment by pattern recognition molecules and incorporate exogenous proteins. After migration into draining lymph node, DCs project many branches to interact with $\mathrm{T}$ cells and $\mathrm{B}$ cells trough their surface MHC complex to initiate and shape the adaptive immune response, which is dependents upon the information from their pattern recognition molecules. But for the indispensable roles of DCs in the adaptive immunity, they are heterogeneous series of subsets derived from several precursors. DCs are further divided into three main populations by steady-state phenotypes: plasmacytoid DCs (pDCs), conventional DCs (cDCs), and monocyte-derived DC (moDC also known as inflammatory DC) $[18,19]$. These three populations are further divided into detailed subsets by their surface antigens. Among these multiple DCs, cDC (CD8+ CD11b- subset and CD103+ CD11b- subset) and mo-DCs shows highest CP efficiency $[20,21]$.

\section{ERAD Utilized Processing of Extracellular Proteins in CP}

Previous studies about CPs found out two major pathways for transports and processing of extracellular proteins; one is the proteasome pathway and another is the endosome/lysosome pathway. The former pathway is also known as the TAP-dependent pathway and the latter pathway is also described as the TAP-independent pathway. But recent researches about graft rejection have shown that the TAP-dependent proteasome pathway is the principal pathway in CP [22].

However in the proteasome pathway, exogenous proteins are transported into the cytosol so as to be processed by the ubiquitin proteasome system, they are not incorporated into the cytosol directly. Exogenous proteins are incorporated into endocytic compartments, such as phagosome or endosome at first. In CPs, endogenous proteins are transported through the lipid bilayer before degradation by the lysosomal protease. This particular activity to transport whole exogenous proteins through lipid bilayerinto the cytosol is eminent in DCs. Other APCs such as macrophages and B cells are below DCs in this specific ability [23]. To this end, DCs are furnished with two appropriate abilities; one is a low proteolytic ability of lysosomal proteases and the other is the high efficiency to transport exogenous proteins into the cytosol. To protect exogenous proteins from lysosomal proteases, DCs shows the low activity of lysosomal proteases [24] by keeping phagosome and endosome/lysosome under alkaline $\mathrm{pH}$ conditions as 7.5$8[25,26]$. In addition, inhibition of lysosomal proteases results in acceleration of CP efficiency in human moDCs or cDC [27-29]. To pass exogenous proteins through cellular membrane, DCs utilize the molecular apparatus of ERAD. ERAD is a universal cellular pathway, which identifies misfolded proteins in ER, transports them into the cytosol trough ER membrane, and finally degrades them by the ubiquitin-proteasome system[30-32]. Inhibition of some of these ERAD-related molecule impaired CP ability strongly indicated the essential roles of ERAD in CP [3335]. But for those important roles of ERAD in $\mathrm{CP}$, recent research indicated that the transport of exogenous proteins into the cytosol is carried out in endocytic compartments and not in ER. ERAD-related molecules are localized to phagosome or endosome [36-38]. In addition, a restricted localization of the Sec61 complex, which is the singular translocon into $\mathrm{CP}$, in ER strongly, inhibited CP [39]. Those results indicate that transports of exogenous proteins out of lipid bilayer was carried out neither in ER nor in the classical endosome, but in endosome with ER resident molecules [39].

\section{A Cellular Compartment for Peptides Loading upon MHC I}

The cellular compartments for the peptide loading on MHC I in CPs are still controversial. Strong amino peptidase activities both in the cytosol and in ER suggest that antigenic peptides are inserted into peptide binding groove soon after processed by ubiquitin proteasome system. In addition, the variations of antigenic peptides upon MHC I is significant for acquired immunity, the peptide loading is carried out with the aid of PLC as DP. These presumptive evidence give us suggestions that the peptide loading in CPs is carried out in above endosome with ER resident molecules. Recent researches support the hypothesis. (i) Observations by optical microscopes and electron microscopes showed that PLC molecules were also localized in endosome and phagosome [34,36$38,40,41]$. (ii) The transportation of MHC I into endocytic compartments was essential for CP [42-44]. (iii) Fully conformed MHC I without antigenic peptide was found in endocytic compartments [45]. (v)CD74 showed an important role in CP by regulating the transport of MHC I into endocytic compartments in DCs [43]. Of late, we purified the sub cellular compartment where exogenous proteins undergo ERAD processing from moDCs derived cell line DC2.4 [46]. In our purified microsome we could detect both ER-resident proteins and endocytic compartment-specific proteins; ER-resident proteins 


\section{Open Access Journal of Pharmaceutical Research}

(Sec61- $\alpha$ and GP96), MHC I and PLC proteins (Calreticulin, ERp57 (PDI), Tapasin, and TAP complex), ERAD-related proteins (CHIP, VCP, and proteasomes), and endosomespecific proteins (Lamp1 and Rab5). Among these molecules, it is note worthy that Lamp1 was detected as both in the precursor form and in the mature form and that fully conformed MHC I were loaded with the pOVA epitope (Figure 1). In addition using our purified compartment, we reconstituted processing of extracellular proteins in vitro [46]. Those results strongly suggest that these purified microsomes correspond to the non-classical endocytic compartment for CPs, which is responsible for both processing of exogenous proteins and peptide loading upon MHC I. We are now attempting in vitro reconstruction of peptide loading using our purified microsome.

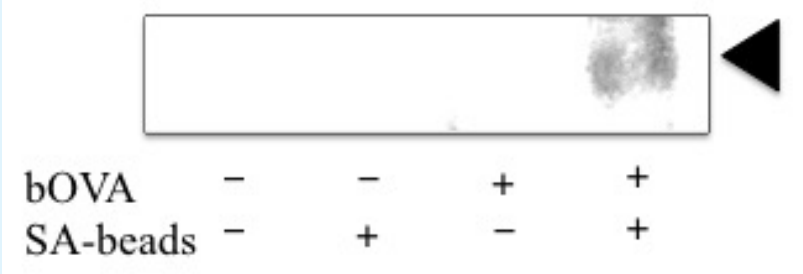

Figure 1: Microsomes with $(+)$ or without $(-)$ prior addition of bOVA were purified with $(+)$ or without (-) SAmagnetic beads as described in our previous article [46]. Proteins $(10 \mu \mathrm{g})$ or corresponding volumes of purified proteins were immuno precipitated by 25D1.16 antibody which specifically recognize $\mathrm{H}^{2} \mathrm{~K}^{\mathrm{b}}$ allele of MHC I loaded with pOV8 epitope. After SDS-PAGE blotting was performed with anti- $\mathrm{H}^{2} \mathrm{~K}^{\mathrm{b}}$ antibody. A triangle in right side indicates the $\mathrm{H}^{2} \mathrm{~K}^{\mathrm{b}}$.

\section{Molecular Mechanisms of Integrations among ER and Endocytic Compartments}

But for the amassed efforts to elucidate the molecular mechanisms of $\mathrm{CP}$, the precious molecular mechanism for the localizations of ER-resident proteins into endocytic compartments remained to be clarified. Recent researches shed a light on this question. (i) SEC22b played a significant role by regulating the fusion of phagosome and ER-Golgi intermediate complex [47]. (ii) Transport of MHC I into endocytic compartments are regulated by Rab22a [48]. (iii) Rab43 is essential in cDCs but not in moDC [49]. (iv) p38 $\alpha$ plays important roles both in CP and cytokines production in CDC [50]. (v) CP efficiency is enhanced by activation of innate immunity [51]. All these results strongly suggest that DCs flexibly integrate membrane transport pathway by responding to environmental conditions for CP.

\section{Concluding Remarks}

But for the important roles of CP in both activation and inactivation of cell mediated immunity, molecular mechanisms for CP are not fully clarified yet. Accumulating findings strongly suggest that $\mathrm{CP}$ is dependent upon the inimitable vesicle transport systems in DCs. So as to integrate innate immunity and adaptive immunity, DCs acquire environmental information by their pattern recognition molecules and shape the most efficient immuno response. DCs equipped several redundant pathways for antigen presentations, which is running alongside with each other, and change vesicle transport system flexibly to fit environmental situations. To apply CP for DC-based immuno therapy, the elucidations of molecular mechanisms of $\mathrm{CP}$ are the issues of importance in researches of immunology field.

\section{References}

1. Van Endert P (2011) Providing ligands for MHC class I molecules. Cell Mol Life Sci 68(9): 1467-1469.

2. Dotiwala F, Mulik S, Polidoro RB, Ansara JA, Burleigh BA, et al. (2016) Killer lymphocytes use granulysin, perforin and granzymes to kill intracellular parasites. Nat Med 22(2): 210-216.

3. Yang D, Torres CM, Bardhan K, Zimmerman $\mathrm{M}$, McGaha TL, et al. (2012) Decitabine and vorinostat cooperate to sensitize colon carcinoma cells to Fas ligand-induced apoptosis in vitro and tumor suppression in vivo. J Immunol 188(9): 4441-4449.

4. McDevitt HO (2000) Discovering the role of the major histocompatibility complex in the immune response. Annu Rev Immunol 18: 1-17.

5. Momburg F, Roelse J, Howard JC, Butcher GW, Hämmerling GJ, et al. (1994) Selectivity of MHCencoded peptide transporters from human, mouse and rat. Nature 367(6464): 648-651.

6. Obst R, Armandola EA, Nijenhuis M, Momburg F, Hammerling GJ (1995) TAP polymorphism does not influence transport of peptide variants in mice and humans. Eur J Immunol 25(8): 2170-2176.

7. Janeway C, Travers $\mathrm{P}$, Walport $\mathrm{M}$, Shlomchik M (2001) Immunobiology: The Immune System in Health and Disease 5th edn. Garland Press, New York.

8. Banchereau J, Steinman RM (1998) Dendritic cells and the control of immunity. Nature 392(6673): 245252. 


\section{Open Access Journal of Pharmaceutical Research}

9. Lanzavecchia A, Sallusto F (2001) Regulation of T cell immunity by dendritic cells. Cell 106(3): 263-266.

10. Joffre OP, Segura E, Savina A, Amigorena S (2012) Cross-presentation by dendritic cells. Nat Rev Immunol 12(8): 557-569.

11. Bevan MJ (1976) Minor $\mathrm{H}$ antigens introduced on $\mathrm{H}-2$ different stimulating cells cross-react at the cytotoxic $\mathrm{T}$ cell level during in vivo priming. J Immunol 117(6): 2233-2238.

12. Ackerman AL, Cresswell P (2004) Cellular mechanisms governing cross-presentation of exogenous antigens. Nat Immunol 5(7): 678-684.

13. Rock KL, Shen L (2005) Cross-presentation: underlying mechanisms and role in immune surveillance. Immunol Rev 207: 166-183.

14. Heath WR, Carbone FR (2001) Cross-presentation in viral immunity and self-tolerance. Nat Rev Immunol 1(2): 126-134.

15. Kurts C, Heath WR, Carbone FR, Allison J, Miller JF, et al. (1996) Constitutive class I-restricted exogenous presentation of self antigens in vivo. J Exp Med 184(3): 923-930.

16. Kurts C, Kosaka H, Carbone FR, Miller JF, Heath WR (1997) Class I-restricted cross-presentation of exogenous self-antigens leads to deletion of autoreactive CD8(+) T cells. J Exp Med 186(2): 239245.

17. Bonifaz L, Bonnyay D, Mahnke K, Rivera M, Nussenzweig MC, et al. (2002) Efficient targeting of protein antigen to the dendritic cell receptor DEC-205 in the steady state leads to antigen presentation on major histocompatibility complex class I products and peripheral CD8+ T cell tolerance. J Exp Med 196(12): 1627-1638.

18. Segura E, Touzot M, Bohineust A, Cappuccio A, Chiocchia G, et al. (2013) Human inflammatory dendritic cells induce Th17 cell differentiation. Immunity 38(2): 336-348.

19. Luber CA, Cox J, Lauterbach H, Fancke B, Selbach M, et al. (2002) Quantitative proteomics reveals subsetspecific viral recognition in dendritic cells. Immunity 32(2): 279-289.

20. Den Haan JM, Lehar SM, Bevan MJ (2000) CD8(+) but not CD8(-) dendritic cells cross-prime cytotoxic T cells in vivo. J Exp Med 192(12): 1685-1696.
21. Markov OV, Mironova NL, Vlasov VV, Zenkova MA (2016) Molecular and Cellular Mechanisms of Antitumor Immune Response Activation by Dendritic Cells. Acta Naturae 8(3): 17-30.

22. Belz GT, Behrens GM, Smith CM, Miller JF, Jones C, et al. (2002) The CD8 alpha(+) dendritic cell is responsible for inducing peripheral self-tolerance to tissue-associated antigens. J Exp Med 196(8): 10991104.

23. Rodriguez A, Regnault A, Kleijmeer M, RicciardiCastagnoli P, Amigorena S (1999) Selective transport of internalized antigens to the cytosol for MHC class I presentation in dendritic cells. Nat Cell Biol 1(6): 362368.

24. Delamarre L, Pack M, Chang H, Mellman I, Trombetta ES (2005) Differential lysosomal proteolysis in antigen-presenting cells determines antigen fate. Science 307(5715): 1630-1634.

25. Savina A, Jancic C, Hugues $S$, Guermonprez $P$, Vargas $\mathrm{P}$, et al. (2006) NOX2 controls phagosomal pH to regulate antigen processing during cross presentation by dendritic cells. Cell 126(1): 205-218.

26. Claus V, Jahraus A, Tjelle T, Berg T, Kirschke H, et al. (1998) Lysosomal enzyme trafficking between phagosomes, endosomes, and lysosomes in J774 macrophages. Enrichment of cathepsin $\mathrm{H}$ in early endosomes. J Biol Chem 273(16): 9842-9851.

27. Chatterjee B, Smed-Sorensen A, Cohn L, Chalouni C, Vandlen R, et al. (2012) Internalization and endosomal degradation of receptor-bound antigens regulate the efficiency of cross presentation by human dendritic cells. Blood 120(10): 2011-2020.

28. Accapezzato D, Visco V, Francavilla V, Molette C, Donato T, et al. (2005) Chloroquine enhances human CD8+ $\mathrm{T}$ cell responses against soluble antigens in vivo. J Exp Med. 202(6): 817-828.

29. Belizaire R, Unanue ER (2009) Targeting proteins to distinct subcellular compartments reveals unique requirements for MHC class I and II presentation. Proc Natl Acad Sci USA 106(41): 17463-17468.

30. Wiertz EJ, Tortorella D, Bogyo M, Yu J, Mothes W, et al. (1996) Sec61-mediated transfer of a membrane protein from the endoplasmic reticulum to the proteasome for destruction. Nature 384(6608): 432438. 


\section{Open Access Journal of Pharmaceutical Research}

31. Hampton RY (2002) ER-associated degradation in protein quality control and cellular regulation. Curr Opin Cell Biol 14(4): 476-482.

32. Tsai B, Ye Y, Rapoport TA (2002) Retro-translocation of proteins from the endoplasmic reticulum into the cytosol. Nat Rev Mol Cell Biol 3(4): 246-255.

33. Ackerman AL, Giodini A, Cresswell P (2006) A role for the endoplasmic reticulum protein retrotranslocation machinery during cross presentation by dendritic cells. Immunity 25(4): 607-617.

34. Imai J, Hasegawa H, Maruya M, Koyasu S, Yahara I (2005) Exogenous antigens are processed through the endoplasmic reticulum-associated degradation (ERAD) in cross-presentation by dendritic cells. Int Immunol 17(1): 45-53.

35. Zehner M, Marschall AL, Bos E, Schloetel JG, Kreer C, et al. (2015) The translocon protein Sec61 mediates antigen transport from endosomes in the cytosol for cross-presentation to CD8(+) T cells. Immunity 42(5): 850-863.

36. Houde M, Bertholet S, Gagnon E, Brunet S, Goyette G, et al. (2003) Phagosomes are competent organelles for antigen cross-presentation. Nature 425(6956): 402-406.

37. Guermonprez P, Saveanu L, Kleijmeer M, Davoust J, Van Endert P, et al. (2003) ER-phagosome fusion defines an MHC class I cross-presentation compartment in dendritic cells. Nature 425(6956): 397-402.

38. Burgdorf S, Scholz C, Kautz A, Tampe R, Kurts C (2008) Spatial and mechanistic separation of crosspresentation and endogenous antigen presentation. Nat Immunol 9(5): 558-566.

39. Zehner M, Marschall AL, Bos E, Schloetel JG, Kreer C, et al. (2015) The translocon protein Sec61 mediates antigen transport from endosomes in the cytosol for cross-presentation to CD8(+) T cells. Immunity 42(5): 850-863.

40. Kovacsovics Bankowski M, Rock KL (1995) A phagosome-to-cytosol pathway for exogenous antigens presented on MHC class I molecules. Science 267(5195): 243-246.

41. Ackerman AL, Kyritsis C, Tampé R, Cresswell P (2003) Early phagosomes in dendritic cells form a cellular compartment sufficient for cross presentation of exogenous antigens. Proc Natl Acad Sci USA 100(22): 12889-12894.

42. Lizée G, Basha G, Tiong J, Julien JP, Tian M, et al. (2003) Control of dendritic cell cross-presentation by the major histocompatibility complex class I cytoplasmic domain. Nat Immunol 4(11): 1065-1073.

43. Basha G, Omilusik K, Chavez-Steenbock A, Reinicke AT, Lack N, et al. (2012) A CD74-dependent MHC class I endolysosomal cross-presentation pathway. Nat Immunol 13(3): 237-245.

44. Merzougui N, Kratzer R, Saveanu L, Van Endert P (2011) A proteasome-dependent, TAP-independent pathway for cross-presentation of phagocytosed antigen. EMBO Rep 12(12):1257-1264.

45. Zagorac GB, Mahmutefendic H, Tomas MI, Kucic N, Le Bouteiller P, et al. (2012) Early endosomal rerouting of major histocompatibility class I conformers. J Cell Physiol 227(7): 2953-2964.

46. Imai J, Otani M, Sakai T, Hatta S (2016) Purification of the subcellular compartment in which exogenous antigens undergo endoplasmic reticulum-associated degradation from dendritic cells. Heliyon 2(9): e00151.

47. Cebrian I, Visentin G, Blanchard N, Jouve M, Bobard A, et al. (2011) Sec22b regulates phagosomal maturation and antigen cross presentation by dendritic cells. Cell 147(6): 1355-1368.

48. Cebrian I, Croce C, Guerrero NA, Blanchard N, Mayorga LS (2016) Rab22a controls MHC-I intracellular trafficking and antigen crosspresentation by dendritic cells. EMBO Rep 17(12): 1753-1765.

49. Kretzer NM, Theisen DJ, Tussiwand R, Briseño CG, Grajales-Reyes GE, et al. (2016) RAB43 facilitates cross-presentation of cell-associated antigens by CD8 $\alpha+$ dendritic cells. J Exp Med 213(13): 2871-2883.

50. Zhou Y, Wu J, Liu C, Guo X, Zhu X, et al. (2016) p38 $\alpha$ has an important role in antigen cross-presentation by dendritic cells. Cell Mol Immunol.

51. Palm NW, Medzhitov R (2009) Pattern recognition receptors and control of adaptive immunity. Immunol Rev 227(1): 221-233. 